Chapter 12

\title{
Cell Death Signaling From the Endoplasmic Reticulum in Soybean
}

\author{
Pedro A.B. Reis and Elizabeth P. B. Fontes \\ Additional information is available at the end of the chapter \\ http://dx.doi.org/10.5772/52711
}

\section{Introduction}

Plants are constantly subjected to adverse environmental conditions, such as extremes of temperature, cold, salinity and drought. As a consequence, plant cells have developed coordinated and integrated mechanisms that respond to these injuries and are immediately activated upon stresses. To cope with the stress, cell signaling pathways are activated and promote up or down regulation of specific genes, which minimize the deleterious effect of stresses within the cell. The endoplasmic reticulum (ER) is a key signaling organelle involved in the activation of cellular stress responses in eukaryotic cells. One such well-characterized signaling event is the unfolded protein response (UPR), which is activated to cope with the disruption of ER homeostasis that results in the accumulation of unfolded or misfolded proteins in the lumen of the organelle. Upon disruption of ER homeostasis, plant cells activate at least two branches of the unfolded protein response (UPR) through IRE1like (Inositol-Requiring Enzyme 1) and ATF6-like (Activating Transcription factor 6) transducers, resulting in the up-regulation of ER-resident molecular chaperones and the activation of the ER-associated degradation protein system. However, if ER stress is sustained, an apoptotic pathway is activated. Persistent ER stress has been shown to trigger both ER-stress specific apoptotic pathways and shared PCD (programmed cell death) signaling pathways elicited by other death stimuli. One plant-specific, ER stress-shared response is the ER and osmotic stress-integrated signaling, which converges on N-rich proteins (NRPs) to transduce a cell death signal. NRP-mediated cell death signaling is a distinct, plant-specific branch of the ER stress pathway that has been shown to integrate the ER and osmotic stress signals into a full response. This ER- and osmotic-stress induced cell death signaling pathway has been uncovered in soybean and constitutes the major focus of this chapter. A second cell death pathway induced by ER stress has been shown to be mediated 
by the $\mathrm{G}$ protein in Arabidopsis, but it remains to be determined whether it operates in soybean as well.

\section{ER stress response: The cytoprotective unfolded protein response}

The ER is a highly dynamic organelle, which mediates several cellular functions, such as the folding and post-translational modification of secretory proteins and protein quality control in addition to maintaining $\mathrm{Ca}^{2+}$ homeostasis (Schröder 2008). The loading of unfolded protein in the lumen of ER for maturation is tightly controlled and dependent on the cellular requirements. Under stress conditions, the folding capacity of the ER can be overloaded causing the accumulation of unfolded proteins and disruption of cellular homeostasis $(\mathrm{Xu}$, Bailly-Maitre \& Reed 2005). To cope with this stress condition, eukaryotic cells evolved a sophisticated signaling mechanism referred to as unfolded protein response (UPR; Malhotra and Kaufman., 2007). In mammalian cells, the UPR is transduced through three distinct ERtransmembrane sensors: PERK (protein kinase RNA-like ER kinase), Ire1 (inositol-requiring enzyme-1) and the basic leucine zipper transcription factor ATF6 (activating transcription factor-6; Ron and Walter, 2007; Malhotra and Kaufman, 2007, Kapoor and Sanyal, 2009). The activation of the UPR allows the ER processing and folding capacities to be balanced with protein loading into the lumen of the organelle under conditions of ER stress (Malhotra and Kaufman, 2007). This balance is achieved by (i) shutting down protein synthesis via PERK activation, (ii) up-regulating the expression of ER-resident processing proteins, such as molecular chaperones and foldases, via activation of Ire1 and ATF6, and (iii) inducing the ERassociated protein degradation (ERAD) machinery, through activation of Ire1, which mediates the targeting and subsequent degradation of unfolded proteins by the proteosome. However, if the ER stress is sustained, multiple apoptotic pathways can be activated in mammalian cells.

In plants, the UPR seems to operate as a bipartite module, as the ER stress signal is transduced through homologs of the Ire1 and ATF6 transducers, but a PERK-mediated branch of the UPR has not been shown (Urade 2009; Chen and Brandizzi., 2012). Two components of the Ire1-mediaded branch of the UPR is known. The first one is the Ire1 ortholog that is represented by two copies in the Arabidopsis genome, Ire1a and Ire1b, and one copy, OsIre1, in the rice genome. Like the mammalian counterpart, plant Ire1 is associated with the ER membrane and exhibits ribonuclease activity and autophosphorylation activities, as shown for Ire1a, Ire1b and OsIre1(Koizumi et al., 2001; Okushima et al., 2002). The second component is ER membrane-associated transcription factor bZIP60. Upon ER stress, bZIP60 mRNA is spliced in an IRE1-mediated process to generate an alternatively spliced transcript that lacks the transmembrane domain-encoding sequences (Liu et al., 2007 e 2008 ; Deng et al., 2011; Nagashima et al., 2011). This splicing leads to the synthesis of a soluble and functional bZIP60 transfactor that can be translocated to the nucleus, where it activates ER stress inducible promoters, such as the BiP3 promoter. Likewise, OsbZIP74 or OsbZIP50 from rice, an ortholog of Arabidopsis AtbZIP60, is regulated through the IRE1-mediated splicing of its 
RNA to render the activation of ER stress-inducible promoters (Hayashi et al., 2011; Lu et al., 2011).

The second branch of UPR in plants mechanistically resembles the ATF6-mediated transduction of the ER stress signal. Upon ER stress, the membrane-associated Arabidopsis ATF6 homologs bZIP17 and bZIP28 are relocated to the Golgi, where their transcriptional domains are proteolytically released from the membrane by SP2 (Tajima et al., 2008: Che et al., 2010). The released bZIP domain of these transfactors is then translocated to the nucleus, where it acts in concert with the heterotrimeric NF-Y complex to activate UPR genes (Liu e Howel., 2010). The NF-Y complex is composed the transcriptional factors NF-YA4, NF-YB3 and NFYC2.

Comprehensive genome-wide evaluations of ER stress-induced changes in gene expression have provided evidence that the UPR operates in a similar fashion in both soybean and Arabidopsis (Irsigler et al., 2007). Inducers of ER stress, such as tunicamycin and AZC, promote the up-regulation of a class of genes that functions in protein folding and ERAD. In the protein folding category, the up-regulated genes include ER-resident molecular chaperones such as BiP, calreticulin, calnexin, and the folding catalyst protein disulfide isomerase (PDI). ERAD-associated genes that are up-regulated by ER stress in soybean include those encoding polyubiquitin, ubiquitin conjugating enzyme, the alpha subunit of the proteasome, CDC48 and Derlin. These genomic analyses suggested that soybean, like Arabidopsis, have evolved at least two different mechanisms that mediate UPR: (i) transcriptional induction of genes encoding chaperones and vesicle trafficking proteins and (ii) upregulation of the ERassociated protein degradation (ERAD) system for rapid disposal of unfolded proteins in the ER as a protective measure.

In addition to the cytoprotective bipartite response to ER stress in plants, two apparently distinct branches of the ER stress-induced pathways have been shown to transduce a cell death signal: (i) the ER membrane associated G $\beta-G \gamma$ heterodimer-mediated signaling events that trigger UPR-associated cell death in Arabidopsis (Wang et al., 2007) and (ii) the ER stressinduced NRP-mediated cell death response that has been uncovered in soybean (Reis and Fontes, 2012).

\section{The ER-stress-induced NRP-mediated cell death response}

NRP-mediated cell death signaling is a distinct, plant-specific branch of the ER stress pathway that has been uncovered in soybean and has been shown to integrate the ER and osmotic stress signals into a full response. This integrative pathway was first identified through genome-wide approaches and expression profiling, which revealed the existence of a modest overlap of the ER and osmotic stress-induced transcriptomes in soybean seedlings treated with PEG (an inducer of osmotic stress) or tunicamycin and AZC (potent inducers of ER stress; Irsigler et al., 2007). The co-regulated genes were first considered to be downstream targets of the integrated pathway based on similar induction kinetics and a synergistic response to the combination of osmotic and ER stress-inducing treatments. Based on these cri- 
teria, the selected downstream components of this ER and osmotic stress responseintegrating pathway encode proteins with diverse roles, such as plant-specific development and cell death (DCD) domain-containing proteins (NRP-A and NRP-B), an ubiquitin-associated (UBA) protein homolog and NAC domain-containing proteins (GmNAC6). Among them, NRP-A and NRP-B were the first ones to be characterized and to show to induce a cell death response when ectopically expressed in tobacco leaves or soybean protoplasts (Costa et al., 2008). As a consequence, the ER and osmotic stress response-integrating pathway has been designated as the NRP-mediated cell death response.

An upstream component of the NRP-mediated cell death response, GmERD15 (Glycine max Early Responsive to Dehydration 15), has been recently identified using one-hybrid screening that targeted the NRP-B promoter in yeast (Alves et al., 2011). GmERD15 is induced by ER and osmotic stress to activate the expression of NRP genes (NRP-A and NRP-B). Up-regulation of NRP-B leads to the induction of an NAC domain-containing protein, GmNAC6, which is a critical mediator of stress-induced cell death in plants (Faria et al., 2011). These components of the ER stress-induced NRP-mediated cell death signaling pathway, GmERD15, NRPs and GmNAC6, have been further characterized.

\subsection{GmERD15 is a ssDNA binding transcriptional activator}

The Early Responsive Dehydration (ERD) genes are rapidly induced in response to water deficit and form a family comprised by ERD1 to ERD16 representatives. The ERD encoded proteins exhibit diverse and heterogeneous biochemical functions and fall into different classes of proteins, such as chloroplast ATP-dependent protease (ERD1), cytosolic HSP70 (ERD2), glutationa-S-transferases (ERD9,ERD11, ERD13) among others (Soitano et al., 2008; Kiyosue et al., 1994; Kiyosue et al., 1993). ERD15 was first identified in Arabidopsis as a hydrophilic protein that possesses a PAM2 domain that interacts with polyA-binding proteins (PABP11; Kiyosue et al., 1994; Kariola et al., 2006)., ERD15 has been shown to function as a negative regulator of the abcisic acid (ABA)-mediated response (Kariola et al., 2006). Overexpression of ERD15 reduces the ABA sensitivity of Arabidopsis, whereas silencing of ERD15 by RNAi promotes hypersensitivity to the hormone. The negative effect of ERD15 on ABA signaling enhances salicylic acid-dependent defense because overexpression of ERD15 was associated with increased resistance to the bacterial necrotroph Erwiniacarotovora, and the enhanced induction of marker genes for systemic acquired resistance. These results are consistent with the observed antagonistic effect of ABA on salicylic acid-mediated defense and may implicate ERD15 as a shared component of these responses.

The soybean GmERD15 homolog has been described as a new ER stress- and osmotic stress-induced transcription factor that binds to the promoter and induces the expression of the NRP-B gene. In fact, GmERD15 was isolated by its capacity to associate stably with the promoter of NRP-B in yeast cells using the one-hybrid system (Alves et al., 2011). The GmERD15 binding site in the NRP-B promoter was mapped to a 12-bp palindromic sequence (511 AGCAnnnnTGCT -500) that resembles binding sites for ssDNA binding proteins, such as NF1C and PBF2 that recognize the sequences -TTGGCnnnnnGCCAA-3' and 5- TGACAnnnnTGTCA-3', respectively (Wang and Kiledjian., 2000). Furthermore, GmERD15 is located in the nu- 
cleus, and chromatin immunoprecipitation (ChIP) assays revealed that it binds to the NRP-B promoter in vivo (Alves et al., 2011). The ectopic expression of GmERD15 in soybean cells activates the NRP-B promoter and induces NRP-B expression. Collectively, these results indicate that GmERD15 functions as an upstream component of the NRP-mediated cell death signaling pathway that is induced by ER stress and osmotic stress

\subsection{NRPs: Molecular and functional characterization}

The N Rich Protein (NRP) gene was first identified by its rapid induction in response to pathogen incompatible interactions in soybean (Ludwig and Tenhaken, 2001). The NRP designation was derived from its high content of asparagine residue, about $25 \%$. NRP is represented in the soybean genome by a small family of three genes: NRP-A, NRP-B and NRP-C. The encoded proteins share a highly conserved development and cell death (DCD) domain at the C-terminal portion in addition to a high content of asparagine residues at their more divergent $\mathrm{N}$ termini. The asparagine rich domain is not well characterized but harbors putative glycosylation and myristoylation sites that may be relevant for function. The DCD domain is found exclusively in plant proteins and it is composed of about 130 amino acid residues, organized into several conserved motifs: FGLP and LFL in the N-treminal region of the domain, PAQV and PLxE at its C-terminus (Tenhaken et al; 2005). DCD domain-containing proteins may be subdivided into four groups, according to the localization of the DCD domain in the primary structure. NRPs belong to the the subgroup I of DCD domaincontaining family of proteins, as their domains are located at the C-terminal portion of the protein (Tenhaken et al; 2005).

NRPs are critical mediators of ER and osmotic stress-induced cell death in soybeans (Costa et al., 2008). The cell death response mediated by NRPs resembles a programmed cell death event. The overexpression of NRPs in soybean protoplasts induces caspase-3-like activity and promotes extensive DNA fragmentation. Furthermore, the transient expression of NRPs in plants causes leaf yellowing, chlorophyll loss, malondialdehyde production, ethylene evolution and the induction of senescence marker genes, which are hallmarks of leaf senescence.

NRPs are up-regulated by ER or osmotic stress but need both stress signals for full induction (Isrigler et al., 2007). This synergistic interaction of both signals upon NRP induction indicates that the ER stress and osmotic stress responses converge at the level of gene expression to potentiate a NRP-mediated cell death response (Costa et al., 2008). NRPs are also upregulated by other abiotic and biotic signals, such as salt stress, oxidative stress and pathogens. Because the NRP-mediated cell death signaling pathway represents a shared response to multiple stress signals in plants, it might permit coordinate adaptive cellular responses under a large array of stress conditions

\subsection{GmNAC6 as a downstream component of the NRP-mediated cell death response}

NAC domain-containing proteins are plant-specific transcriptional factors that are expressed in several tissues and developmental stages. The NAC transfactors are organized into a general structure that consists of a highly conserved N-terminal domain involved in 
DNA binding (called NAC domain) and a C-terminal region highly divergent in sequence and length that functions as the activation domain. The NAC domain was derived from comparison of consensus sequences among NAM from Petunia, ATAF1/2 and CUC2 from Arabidopsis (Souer et al., 1996.). It comprises nearly 160 amino acid residues, divided into five subdomains (A-E) exhibiting a negative net charge and a nuclear localization signal (Xie et al., 1999; Seoet al., 2008). The subdomains A, C and D are conserved among plant species whereas B and E subdomains are variable (Ooka et al., 2003). The C- terminus harbors a protein-protein interaction domain in some NAC-containing proteins while a transmembrane domain is present in other transcriptional factors (Seo et al., 2008). Therefore, the NAC family is comprised by both soluble, nuclear transactivators and membrane proteins.

The members of the NAC gene family are involved in a variety of developmental events and defense responses, such as shoot apical meristem formation and maintenance (SAM; Aida et al., 1997; Souer et al.,1996; Weir et al., 2004), hormone signaling (Fujita et al., 2004; Xie et al., 2000), response to pathogen infection (Ren et al., 2000; Selth et al., 2005; Xie et al., 1999), leaf senescence (John et al., 1997) and response to different abiotic stresses (Hegedus et al., 2003; Tran et al.,2004).

The soybean NAC family is comprised by 180 putative sequences of NAC domain-containing proteins, which display different expression profiles in response to distinct environmental stress conditions and developmental signals (Mochida et al.,2009; Mochida et al., 2010; Wang et al., 2010). Frequently, the stress-induced expression profile of the soybean NAC genes reflects the functional profile of the encoded protein (Pinheiro et al., 2009). GmNAC6 was identified by its synergistic induction in response to a combined treatment of inducers of osmotic stress (polyethylene glycol) and ER stress (tunicamycin) and was functionally linked to the NRP-mediated cell death response (Faria et al., 2011). Transient expression of GmNAC6 promotes cell death and hypersensitive-like responses in planta. GmNAC6 and NRPs also share overlapping responses to biotic signals, but the induction of NRPs peaks before the increased accumulation of GmNAC6 transcripts. Consistent with the delayed kinetics of GmNAC6 induction, increased levels of NRP-A and NRP-B transcripts induce promoter activation and the expression of the GmNAC6 gene. Therefore, GmNAC6 is biochemical and functionally linked to the ER stress- and osmotic stress-integrating cell death response, in which it acts downstream of the NRPs.

GmNAC6 encodes a 33kDa protein that belongs to the TERN (Tobacco elicitor-responsive gene encoding NAC domain protein) group of the NAC family, which is induced by elicitors of the pathogen response (Ooka et al.,2003). Likewise, GmNAC6 is induced by the pathogenic bacteria Pseudomonas syringaepatovar tomato which elicits an incompatible interaction in soybean and by cell wall-degrading enzymes, which mimic bacterial pathogen attack. Like GmNAC6, the other components of the ER- and osmotic-stress induced cell death signaling pathway, GmERD15 and NRPs, are also induced by other biotic and abiotic signals, such drought and pathogen incompatible interactions. Therefore, the activation of the NRPmediated senescence-like response is not specific to ER stress or osmotic stress but is, rather, a shared branch of general environmental adaptive pathways. 


\section{Acknowledgement}

This research was supported by the Brazilian Government Agencies CNPq grants 559602/2009-0, 573600/2008-2 and 470878/2006-1 (to E.P.B.F.), the FAPEMIG grant CBBAPQ-00070-09, and the FINEP grant 01.09.0625.00 (to E.P.B.F.). P.A.B.R. is supported by CNPq graduate fellowships.

\section{Author details}

Pedro A.B. Reis and Elizabeth P. B. Fontes

Departamento de Bioquímica e Biologia Molecular/ Bioagro, Universidade Federal de Viçosa, Viçosa, MG, Brazil

\section{References}

[1] Aida, M., Ishida, T., Fukaki, H., Fujisawa, H., \& Tasaka, M. (1997). Genes involved in organ separation in Arabidopsis, an analysis of the cup-shaped cotyledon mutant. The Plant Cell, 0153-2298X, 9, 841-857.

[2] Alves, M. S., Reis, P. A. B., Dadalto, S. P., Faria, J. A. Q. A., Fontes, E. P. B., \& Fietto, L. G. (2010). A novel transcription factor, early responsive to dehydration 15, connects ER stress with an osmotic stress-induced cell death signal. Journal of Biological Chem. istry, 0108-3351X, 286, 20020-20030.

[3] Che, P.; Bussell, J.D.; Zhou, W.; Estavillo, G.M.; Pogson, B.J.; Smith, S.M. (2010) Signaling from the Endoplasmic Reticulum Activates Brassinosteroid Signaling and Promotes Acclimation to Stress in Arabidopsis. Science Signaling, 3: ra69, ISSN 1945-0877

[4] Chen, Y., \& Brandizzi, F. (2012). AtIRE1A/AtIRE1B and AGB1 independently control two essential unfolded protein response pathways in Arabidopsis. The Plant Journal0136-5313X, 69, 266-277.

[5] Costa, M. D. L., Reis, P. A. B., Valente, M. A. S., Irsigler, A. S. T., Carvalho, C. M., Loureiro, M. E., Aragaão, F. J. L., Boston, R. S., Fietto, L. G. ., \& Fontes, E. P. B. (2008). A new branch of endoplasmic reticulum stress signaling and the osmotic signal converge on plant-specific asparagine-rich proteins to promote cell death. Journal of Biological Chemistry0108-3351X, 20209-20219.

[6] Deng, Y., Humbert, S., Liu, J. X., Srivastava, R. J., Rothstein, J. S., \& Howell, S. H. (2011). Heat induces the splicing by IRE1 of a mRNA encoding a transcription factor involved in the unfolded protein response in Arabidopsis. Proceedings of the National Academy of Sciences, 1091-6490, 108, 7247-7252. 
[7] Faria, J. A. Q. A., Reis, P. A. B., Reis, M. T. B., Rosado, G. L., Pinheiro, G. L., Mendes, G. C., \& Fontes, E. P. B. (2011). The NAC domain-containing protein, GmNAC6, is a downstream component of the ER stress- and osmotic stress-induced NRP-mediated cell-death signaling pathway. BMC Plant Biology, 11: 129, 1471-2229, 1471-2229.

[8] Fujita, M., Fujita, Y., Maruyama, K., Seki, Motoaki., Hiratsu, K., Ohme-Takagi, M., Tran-S, L., , P., Yamaguchi-Shinozaki, K., \& Shinozaki, K. (2004). A dehydration-induced NAC protein, RD26, is involved in a novel ABA-dependent stress-signaling pathway. The Plant Journal0136-5313X, 39, 863-876.

[9] Hayashy, S., Wakasa, Y., Takahashi, H., Kawakatsu, T., \& Takaiwa, F. (2012). Signal transduction by IRE1-mediated splicing of bZIP50 and other stress sensors in the endoplasmic reticulum stress response of rice. The Plant Journal, 0136-5313X, 69, 946-956.

[10] Hegedus, D.., Yu, M., Baldwin, D., Gruber, M., Sharpe, A., Parkin, I., Whitwill, S., \& Lydiate, D. . (2003). Molecular characterization of Brassica napus NAC domain transcriptional activators induced in response to biotic and abiotic stress. Plant Molecular Biology, 383-397, 1572-9818.

[11] Irsigler, A. S. T., Costa, M. D. L., Zhang, P., Reis, P. A. B., Dewey, R. E., Boston, R. S. ., \& Fontes, E. P. B. (2007). Expression profiling on soybean leaves reveals integration of ER- and osmotic-stress pathways. BMC Genomics, 1471-2164, 1471-2164.

[12] John, I., Hackett, R., Cooper, W., Drake, R., Farrell, A., \& Grierson, D. (1997). Cloning and characterization of tomato leaf senescence-related cDNAs. Plant Molecular Biolo$g y, 33,641-651,1572-9818$.

[13] Kapoor, A., \& Sanyal, A. J. (2009). Endoplasmic Reticulum Stress and the Unfolded Protein Response. Clinics in Liver Disease, 581-590, 1089-3261.

[14] Kariola, T., Brader, G., Helenius, E., Li, J., Heino, P., \& Palva, E. T. (2006). EARLY RESPONSIVE to DEHYDRATION 15, a negative regulator of abscisic acid responses in Arabidopsis. Plant Physiology, 1532-2548, 142, 1559-73.

[15] Kiyosue, T., Yamaguchi-Shinozaki, K., \& Shinozaki, K. (1994). Cloning of cDNAs for genes that are early-responsive to dehydration stress (ERDs) in Arabidopsis thaliana L.: identification of three ERDs as HSP cognate genes. Plant Molecular Biology, 25, 791-8, 1572-9818.

[16] Kiyosue, T., Yamaguchi-Shinozaki, K., \& Shinozaki, K. (1993). Characterization of two cDNAs (ERD11 and ERD13) for dehydrationinducible genes that encode putative glutathione S-transferases in Arabidopsis thaliana L. FEBS Letters, 0014-5793, 335, 189-92.

[17] Koizumi, N., Martinez, I. M., Kimata, Y., Kohno, K., Sano, H., \& Chrispeels, M. J. (2001). Molecular characterization of two Arabidopsis Ire1 homologs, endoplasmic reticulum-located transmembrane protein kinases. Plant Physiology, 1532-2548, 127, 949-962. 
[18] Liu, J. X., Srivastava, R. ., , P. ., \& Howell, S. H. (2007). Salt stress responses in Arabidopsis utilize a signal transduction pathway related to endoplasmic reticulum stress signaling. The Plant Journal, 0136-5313X, 51, 897-909.

[19] Liu, J. X., Srivastava, R., \& Howell, S. H. (2008). Stress-induced expression of an activated form of AtbZIP17 provides protection from salt stress in Arabidopsis. PlantCell \& Environment. 1365-3040, 31, 1735-1743.

[20] Liu, J. X., \& Howell, S. H. (2010). Endoplasmic Reticulum Protein Quality Control and Its Relationship to Environmental Stress Responses in Plants. The Plant Cell, 0153-2298X, 22, 1-13.

[21] Lu-J, S., Yang-T, Z., Sun, L., Sun, L., Song-T, Z., \& Liu-X, J. (2011). Conservation of IRE1 -Regulated bZIP74 mRNA Unconventional Splicing in Rice (Oryza sativa L.) Involved in ER Stress Responses. Molecular Plant, 1752-9867.

[22] Ludwig, A. A., Tenhaken, R., \& (2001, . (2001). A new cell wall located N-rich protein is strongly induced during the hypersensitive response in Glycine max L. European Journal of Plant Pathology, 107, 323-336, 1573-8469.

[23] Malhotra, J. D. ., \& Kaufman, R. J. (2007). The Endoplasmic Reticulum and the Unfolded Protein Response. Semininars in Cell and Developmental Biology, 1084-9521, $18,716-73$.

[24] Mochida, K., Yoshida, T., Sakurai, T., Yamaguchi-Shinozaki, K., Shinozaki, K., \& Tran, L. S. (2009). In silico analysis of transcription factor repertoire and prediction of stress responsive transcription factors in soybean. DNA Research., 1756-1663, 16, 353-69.

[25] Mochida, K., Yoshida, T., Sakurai, T., Yamaguchi-Shinozaki, K., Shinozaki, K., \& Tran, L. S. (2010a). LegumeTFDB: an integrative database of Glycine max, Lotus japonicus and Medicagotruncatula transcription factors. Bioinformatics, 26, 290-1, 1460-2059.

[26] Nagashima, Y., Mishiba, K., Suzuki, E., Shimada, Y., Iwata, Y., \& Koizumi, N. (2011). Arabidopsis IRE1 catalyses unconventional splicing ofbZIP60 mRNA to produce the active transcription factor. Scientific Reports, 1:29, 2045-2322, 2045-2322.

[27] Okushima, Y., Koizumi, N., Yamaguchi, Y., Kimata, ., Kohno, K., \& Sano, H. (2002). Isolation and characterization of a putative transducer of endoplasmic reticulum stress in Oryza sativa. Plant Cell Physiology, 1471-9053, 43, 532-539.

[28] Ooka, H., Satoh, K., Doi, K., et al., et al. (2003). Comprehensive analysis of NAC family genes in Oryza sativa and Arabidopsis thaliana. DNA Research. 10; 1756-1663, 239-247.

[29] Pinheiro, G. L., Marques, C. S., Costa, M. D. B. L., Reis, P. A. B., Alves, M. S., Carvalho, C. M., Fietto, L. G. ., \& Fontes, E. P. B. (2009). Complete inventory of soybean NAC transcription factors: Sequence conservation and expression analysis uncover their distinct roles in stress response. Gene, 444, 10-23, 0378-1119. 
[30] Reis, P.A.B and Fontes, E.P.B.(2012). N-rich protein (NRP)-mediated cell death signaling: a new branch of the ER stress response with implications for plant biotechnology. Plant Signaling \& Behavior. 1559-2316, 7

[31] Ren, T., Qu, F., \& Morris, T. J. (2000). HRT gene function requires interaction between a NAC protein and viral capsid protein to confer resistance to turnip crinkle virus. The Plant Cell 0153-2298X, 12, 1917-1926.

[32] Ron, D., \& Walter, P. (2007). Signal integration in the endoplasmic reticulum unfolded protein response. Nature ReviewsMolecular Cell Biology 1471-0072, 8, 519-529.

[33] Schröder, M. (2008). Endoplasmic reticulum stress responses. Cellular and Molecular Life Sciences0142-0682X, 65, 862-894.

[34] Selth, L. A., Dogra, S. C., Rasheed, M. S., Healy, H., Randles, J. W., \& Rezaian, M. A. (2005). A NAC domain protein interacts with tomato leaf curl virus replication accessory protein and enhances viral replication. The Plant Cell 0153-2298X, 17, 311-325.

[35] Seo, P. J., Kim, S. G., \& Park, C. M. (2008). Membrane bound transcription factors in plants. Trends in Plant Science1360-1385, 13, 550-6.

[36] Souer, E., van Houwelingen, A., Kloos, D., Mol, J., \& Koes, R. (1996). The no apical meristem gene of Petunia is required for pattern formation in embryos and flowers and is expressed at meristem and primordial boundaries. Cell, 0092-8674, 85, 159-70.

[37] Tajima, H., Iwata, Y., Iwano, M., Takayama, S., \& Koizumi, N. (2008). Identification ofan Arabidopsis transmembranebZIP transcription factor involved in the endoplasmicreticulum stress response. Biochemical and Biophysical Research Communications, 0000-6291X, 374, 242-247.

[38] Tenhaken, R., Doerks, T., \& Bork, P. (2005). DCD- a novel plant specific domain in proteins involved in development and programmed cell death. BMC Bioinformatics 6:169, 1471-2105, 1471-2105.

[39] Tran, L. S., Nakashima, K., Sakuma, Y., et al. (2004). Isolation and functional analysis of Arabidopsis stress-inducible NAC transcription factors that bind to a drought-responsive cis-element in the early responsive to dehydration stress 1 promoter. The Plant Cell0153-2298X, 16, 2481-2498.

[40] Udvardi, M. K., Kakar, K., Wandrey, M., et al. (2007). Legume transcription factors: global regulators of plant development and response to the environment. Plant Physiology, 144, 538-49, 1532-2548.

[41] Wang, S., Narendra, S., \& Fedoroff, N. (2007). Heterotrimeric G protein signaling in the Arabidopsis unfolded protein response. Proceedings of the National Academy of Sciences, ISSN 1091-6490, 104, 3817-3822.

[42] Wang, Z., \& Kiledjian, M. (2000). The Poly(A)-Binding Protein and an mRNA Stability Protein 
[43] Jointly Regulate an Endoribonuclease Activity.Molecular and Cellular Biology, 1098-5549, 20, 6334-6341.

[44] Wang, Z., Libault, M., Joshi, T., et al. (2010). SoyDB: a knowledge database of soybean transcription factors. BMC Plant Biology, 10: 14, 1471-2229, 1471-2229.

[45] Weir, I., Lu, J., Cook, H., Causier, B., Schwarz-Sommer, Z., \& Davies, B. (2004). CUPULIFORMIS establishes lateral organ boundaries in Antirrhinum. Development, 131, 915-922, 1011-6370.

[46] Xie, Q., Sanz-Burgos, A. P., Guo, H., Garcia, J. A., \& Gutierrez, C. (1999). GRAB proteins, novelmembers of the NAC domain family, isolated by their interaction with a geminivirus protein. Plant Molecular Biology. 1572-9818, 39, 647-656.

[47] Xu, C., Bailly-Maitre, B., \& Reed, J. C. (2005). Endoplasmic reticulum stress: cell life and death decisions. Journal of Clinical Investigation. 0021-9738, 115, 2656-2664. 
